\title{
Hybrid Heat Capacity - Moving Slab Laser Concept
}

Eddy A. Stappaerts

April 2002

U.S. Department of Energy

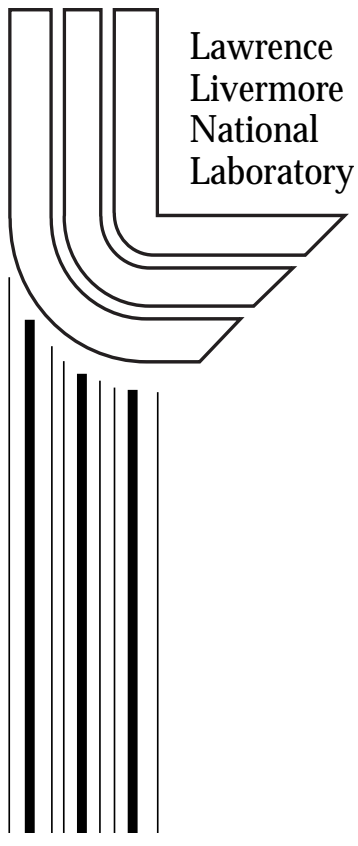




\section{DISCLAIMER}

This document was prepared as an account of work sponsored by an agency of the United States Government. Neither the United States Government nor the University of California nor any of their employees, makes any warranty, express or implied, or assumes any legal liability or responsibility for the accuracy, completeness, or usefulness of any information, apparatus, product, or process disclosed, or represents that its use would not infringe privately owned rights. Reference herein to any specific commercial product, process, or service by trade name, trademark, manufacturer, or otherwise, does not necessarily constitute or imply its endorsement, recommendation, or favoring by the United States Government or the University of California. The views and opinions of authors expressed herein do not necessarily state or reflect those of the United States Government or the University of California, and shall not be used for advertising or product endorsement purposes.

This work was performed under the auspices of the U. S. Department of Energy by the University of California, Lawrence Livermore National Laboratory under Contract No. W-7405Eng-48.

This report has been reproduced

directly from the best available copy.

Available to DOE and DOE contractors from the

Office of Scientific and Technical Information

P.O. Box 62, Oak Ridge, TN 37831

Prices available from (423) 576-8401

http://apollo.osti.gov/bridge/

Available to the public from the

National Technical Information Service

U.S. Department of Commerce 5285 Port Royal Rd., Springfield, VA 22161 http://www.ntis.gov/

OR

Lawrence Livermore National Laboratory Technical Information Department's Digital Library http://www.llnl.gov/tid/Library.html 


\section{Hybrid Heat Capacity - Moving Slab Laser Concept}

Eddy A. Stappaerts

April 2002 


\section{Table of Contents}

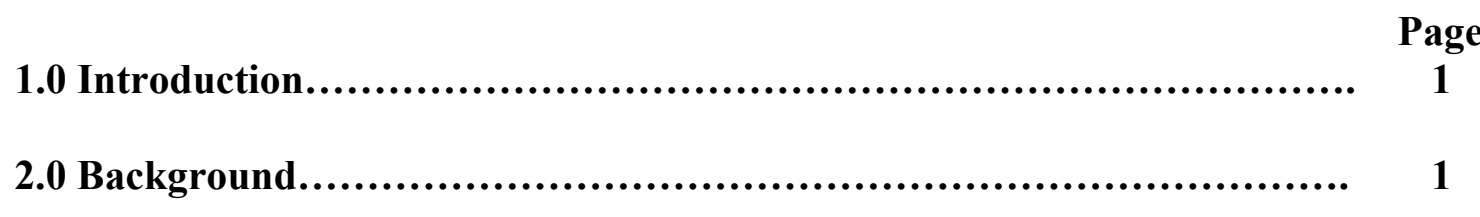

3.0 Concept Description.................................................... $\quad 2$

4.0 Design Study........................................................... 5

4.1 Mechanical design................................................... 6

4.1.1 Concept-1: Translated disk matrix........................... 7

4.1.2 Concept-2: Swing arm..................................... 8

4.1.3 Concept-3: "Slide projector"'................................ 10

4.2 Electrical design.................................................. 13

5.0 Power Scaling......................................................... 13

6.0 Conclusions............................................................ 14

7.0 Acknowledgements..................................................... 14

8.0 References.................................................................... 15 


\subsection{Introduction}

A hybrid configuration of a heat capacity laser (HCL) and a moving slab laser (MSL) has been studied. Multiple volumes of solid-state laser material are sequentially diodepumped and their energy extracted. When a volume reaches a maximum temperature after a "sub-magazine depth", it is moved out of the pumping region into a cooling region, and a new volume is introduced. The total magazine depth equals the submagazine depth times the number of volumes. The design parameters are chosen to provide high duty factor operation, resulting in effective use of the diode arrays. The concept significantly reduces diode array cost over conventional heat capacity lasers, and it is considered enabling for many potential applications.

A conceptual design study of the hybrid configuration has been carried out. Three concepts were evaluated using CAD tools. The concepts are described and their relative merits discussed.

Because of reduced disk size and diode cost, the hybrid concept may allow scaling to average powers on the order of $0.5 \mathrm{MW} /$ module using crystal sizes on the order of $10 \mathrm{~cm}$. A representative design is described.

\subsection{Background}

Heat capacity lasers ${ }^{1,2}$ and moving-slab lasers ${ }^{3,4}$ provide two approaches for scaling solid-state lasers to high average powers. With HCLs, the pumping/lasing and cooling phases are sequential. With MSLs pumping, lasing and cooling are simultaneous, and these lasers can be operated in a CW mode.

The duration of the pumping/lasing phase of a HCL is called the magazine depth. During this time the active medium temperature increases to a maximum value set by lower level bottlenecking and/or engineering constraints. When this temperature is reached pumping/lasing are stopped, and the temperature is reset using passive or active cooling. The pumping/lasing-cooling cycle can then be repeated.

HCLs are typically operated in a pulsed mode. The duty factor is the ratio of the pulse length and the sum of the pulse length and the inter-pulse time. Due to fundamental design constraints, the duty factor is typically low, resulting in sub-optimum use of the diode arrays. Peak power must be sized for efficient HCL operation, but this power is only utilized a fraction of the time. Since diode cost is proportional to peak-power, this results in a cost penalty inversely proportional to duty factor. LLNL has recently demonstrated continuous, microchannel-cooled, diode arrays with irradiances up to 1.5 $\mathrm{kW} / \mathrm{cm}^{2}{ }^{5}$, sufficient to pump HCLs without focusing optics.

With MSLs, the active medium temperature rise is limited by moving a volume of laser material, by translation and/or rotation, in front of a pumping source. This source illuminates only part of the volume at a given time, while the entire volume, or most of the volume, is being cooled continuously. 
The hybrid concept will be explained starting from the HCL configuration of Figure 1, but the conclusions are independent of the pumping and extraction geometry. The laser has $\mathrm{M}$ disks that are pumped longitudinally from both sides.

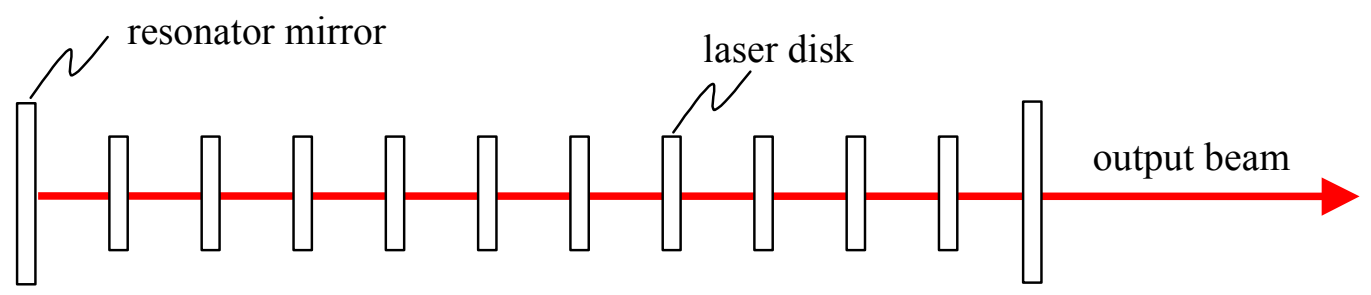

Figure 1: Conventional HCL configuration. The disks are pumped longitudinally through the large crystal faces.

\subsection{Concept Description}

HCLs are described by Equations (1)-(4) for the number of laser disks, the diode array duty factor, $\delta$, the diode array area, $\mathrm{A}$, and the diode array cost, $\mathrm{C}_{\mathrm{d}}$. Requirements are the average output power, $\mathrm{P}_{\mathrm{av}}$, and magazine depth, $\tau_{\mathrm{mag}}$. Material-dependent parameters are the pump photon energy, $h v_{p}$, the stimulated emission cross-section, $\sigma$, the upper-level lifetime, $\tau_{\mathrm{u}}$, the quantum efficiency $\eta_{\mathrm{q}}$, the material density, $\rho$, and the specific heat, $\mathrm{c}_{\mathrm{p}}$. Design parameters to be optimized are the diode array intensity, $\mathrm{I}_{\mathrm{d}}$, unsaturated singlepass gain, $\mathrm{G}$, disk thickness, $\mathrm{t}$, and disk temperature swing, $\Delta \mathrm{T}$. These parameters are constrained by the available diode array irradiance, the gain-to-loss ratio required for efficient laser extraction, the required cooling rate, and the temperature at which the extraction efficiency decreases due to reduced gain.

$$
\begin{aligned}
& M=\frac{h v_{p} G}{2 \sigma I_{d} \eta_{d} \eta_{a} \tau_{u}} \sim \frac{G}{I_{d}} \\
& \delta=\frac{\rho c_{p} t \Delta T}{2 I_{d} \eta_{d} \eta_{a}\left(1-\eta_{q}\right) \tau_{\text {mag }}} \sim \frac{t \Delta T}{I_{d}} \frac{1}{\tau_{\text {mag }}} \\
& A=\frac{P_{\text {out }}}{2 I_{d} \eta_{d} \eta_{a} \eta_{q} \eta_{\text {ext }} \delta M} \sim \frac{1}{I_{d} \delta M} P_{\text {out }} \sim \frac{1}{t \Delta T M} P_{\text {out }} \tau_{\text {mag }} \\
& C_{d}=\frac{P_{\text {out }} c_{W}}{\eta_{d} \eta_{a} \eta_{q} \eta_{\text {ext }} \delta} \sim \frac{P_{\text {out }} c_{W}}{\delta} \sim \frac{I_{d}}{t \Delta T} P_{\text {out }} \tau_{\text {mag }}
\end{aligned}
$$

Diode light delivery efficiency, $\eta_{\mathrm{d}}$, and disk absorption efficiency, $\eta_{\mathrm{a}}$, must be maximized and are near-unity for optimized designs. The extraction efficiency, $\eta_{\text {ext }}$, is determined by the gain-to-loss ratio and, therefore, the pumping intensity. It is typically 
in the $40-80 \%$ range, but its exact value is not important for the present discussion. The diode array cost per peak-Watt is $\mathrm{c}_{\mathrm{w}}$. Equations (1) and (3) are derived from the conventional expressions for laser gain and output power, respectively. Equation (2) is the thermodynamic equation, $\mathrm{Q}=\mathrm{mc} \Delta \mathrm{T}$, where $\mathrm{m}=\rho \mathrm{At}$. Equation (4) relates total diode array cost to array area and pumping intensity.

According to (2) and (4), the duty factor is maximized, and cost minimized, by maximizing disk thickness and temperature swing, and minimizing pumping intensity. Diode cost does not depend explicitly on $G$, with higher $G$ values increasing the number of disks.

A representative HCL design example follows. The requirement is an average power of $40 \mathrm{~kW}$ for $10 \mathrm{~s}$. The laser medium is Nd:GGG. The laser is operated in a pulsed mode, with pulse lengths of $0.5 \mathrm{~ms}$. Material parameters are $\lambda_{\mathrm{p}}=0.805 \mu \mathrm{m}, \lambda_{\mathrm{e}}=1.06 \mu \mathrm{m}, \sigma=$ $2^{*} 10^{-19} \mathrm{~cm}^{2},{ }^{6} \tau_{\mathrm{u}}=270 \mu \mathrm{s},{ }^{7} \rho=7.09 \mathrm{~g} / \mathrm{cm}^{3}{ }^{7}$, and $\mathrm{c}=0.38 \mathrm{~J} / \mathrm{g} / \mathrm{K} .{ }^{7}$ Table 1 , left, shows the laser design parameters obtained from (1)-(4) for $\mathrm{I}_{\mathrm{d}}=1 \mathrm{~kW} / \mathrm{cm}^{2}, \mathrm{t}=1.5 \mathrm{~cm}, \Delta \mathrm{T}=100 \mathrm{C}$, $\mathrm{G}=3, \eta_{\mathrm{d}} \eta_{\mathrm{a}}=0.85, \eta_{\mathrm{q}}=0.76$, and $\eta_{\mathrm{ext}}=0.60$.

The hybrid concept is illustrated in Figure 2. A volume of active medium is pumped at high duty factor until the maximum disk temperature is reached. Pumping is then stopped, the heated material moved out of the pumping region into a cooling region, and a new volume of cool active medium introduced for pumping/lasing. The magazine depth for the individual volumes is short because of the high duty factor. Table 1, right, shows design parameters derived using (1)-(4), for a magazine depth of $10 \mathrm{~s}$ made up of five "sub-magazines" of $2 \mathrm{~s}$ duration, each. The repetition-rate and duty factor for the hybrid HCL-MSL are a factor of five higher than for the HCL, and the diode area and pulse energy are reduced correspondingly.

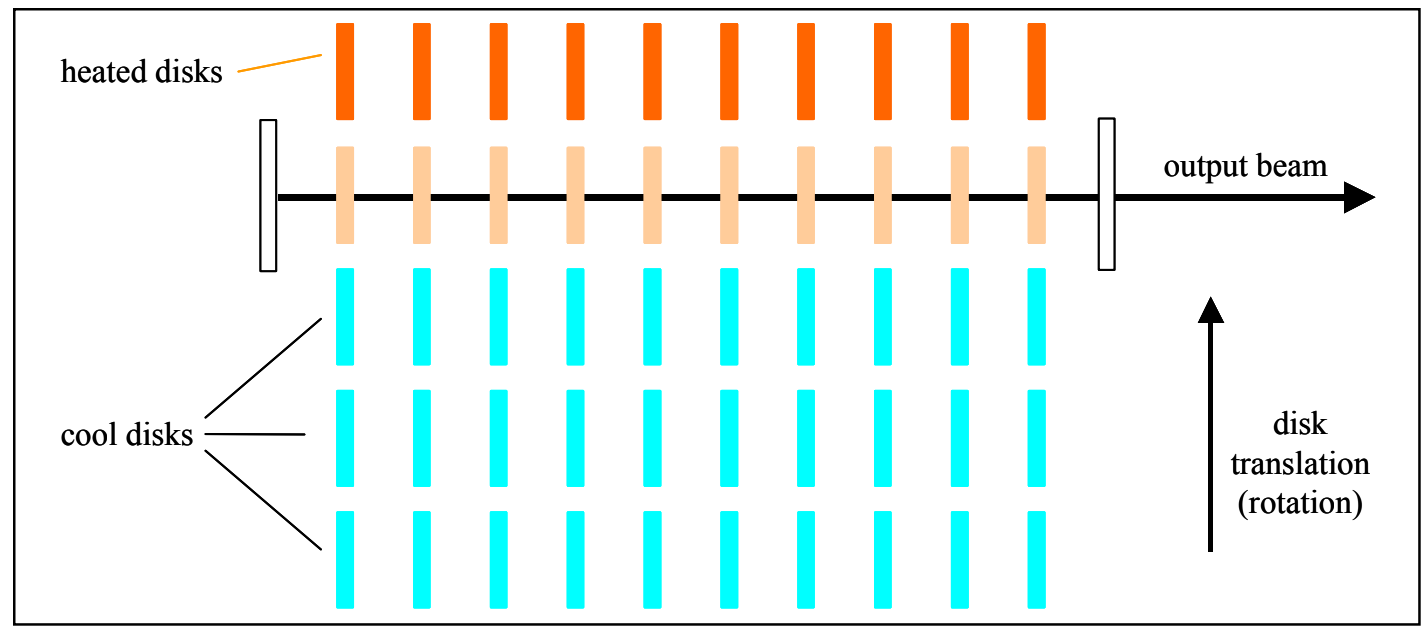

Figure 2: Hybrid HCL-MSL configuration. Sets of cool disks are moved into a resonator, diode-pumped, and moved into a cooling region after a maximum temperature is reached. 
Table 1: Representative parameters for $40 \mathrm{~kW}, 10 \mathrm{~s}$ (0.4 MJ) HCL and hybrid HCL-MSL

\begin{tabular}{|l|c|c|}
\hline Parameter & HCL & Hybrid HCL-MSL \\
\hline Magazine depth (s) & 10 & $2 \times 5$ \\
\hline Pulse energy $(* \mathrm{~J})$ & 200 & 40 \\
\hline Repetition-rate $(\mathrm{Hz})$ & 200 & 1000 \\
\hline Duty factor $(\%)$ & 10 & 50 \\
\hline Number of disks & 10 & 10 \\
\hline Disk thickness & 1.5 & 1.5 \\
\hline Disk width, height $(\mathrm{cm})$ & $7 \times 7$ & $3.1 \times 3.1$ \\
\hline Total disk mass $(\mathrm{kg})$ & 5.5 & $1.1 \times 5$ \\
\hline Gain along diagonal & $\mathrm{e}^{3.6}$ & $\mathrm{e}^{1.6}$ \\
\hline
\end{tabular}

The hybrid concept has additional attractive features. Disk size is smaller than for a conventional HCL, reducing the required crystal boule size. The small-signal gain along a disk diagonal is reduced, simplifying edge claddings for parasitics suppression. Size and cost of the electrical power subsystem are reduced due to the higher duty factor. Since the two designs are thermally equivalent, the size and cost of this subsystem is similar.

Figure 3 shows duty factor and disk size as a function of the number of sub-magazines for the above laser parameters. Total diode array cost, for $\mathrm{c}_{\mathrm{W}}=1 \$ /$ peak-W, decreases from $\$ 1.0 \mathrm{M}$ for a HCL to $\$ 200 \mathrm{k}$ for a hybrid HCL-MSL with $5 \mathrm{sub}$-magazines. This cost reduction is considered enabling for many potential applications.

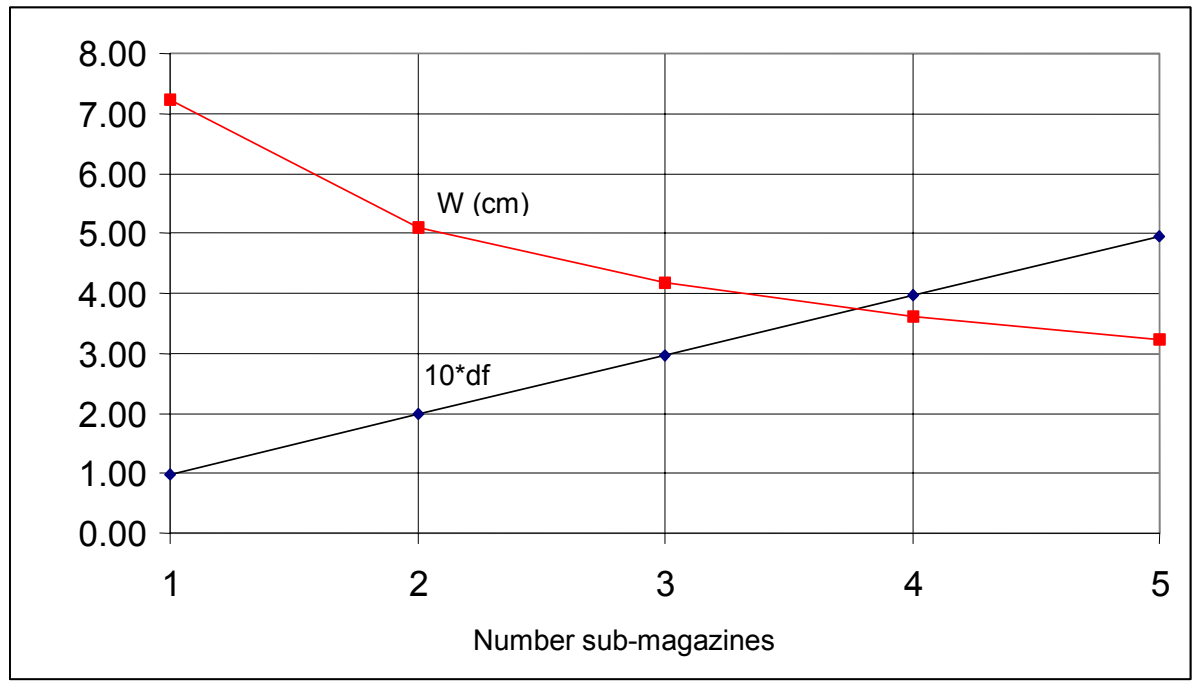

Figure 3: Hybrid HCL-MSL duty factor and slab size vs. number of sub-magazines

Active cooling can be performed in two ways. A first possibility is to exhaust all submagazines followed by simultaneous cooling of all disks. Alternately, cooling of a set of heated disks can be started immediately after they are moved out of the pumping region. 
For a sufficient number of sub-magazines, continuous operation for a limited time is possible. True $\mathrm{CW}$ operation is possible if, in addition, the disk cool-down time equals the total magazine depth.

\subsection{Design Study}

A design study was carried out to evaluate the practical feasibility of the hybrid concept. The study emphasis was on mechanical design, but key electrical design issues were briefly addressed as well.

Design parameters for HCLs and Hybrid HCL-MSLs are shown in Table 4.1. The parameters are not optimized, but they provide a realistic comparison of the concepts.

The following parameters were held fixed: total magazine depth $=10 \mathrm{~s}$, pulse length $=1$ $\mathrm{ms}$, number of disks $=10$, disk thickness $=2 \mathrm{~cm}$, cladding thickness $=1 \mathrm{~cm}$.

Table 4.1 Design Examples

\begin{tabular}{|l|c|c|c|c|c|c|}
\hline Output power (kW) & 30 & 30 & 100 & 100 & 100 & 100 \\
\hline Number sub-magazines & 1 & 5 & 1 & 5 & 1 & 5 \\
\hline Sub-magazine depth (s) & 10 & 2 & 10 & 2 & 10 & 2 \\
\hline $\mathrm{I}_{\text {diode }}\left(\mathrm{kW} / \mathrm{cm}^{2}\right)$ & 1.2 & 1.2 & 1.2 & 1.2 & 2.4 & 2.4 \\
Disk temperature rise (C) & 100 & 100 & 100 & 100 & $160^{+}$ & $160^{+}$ \\
\hline Pulse energy (J) & 338 & 68 & 1125 & 225 & 703 & 141 \\
Rep-rate (Hz) & 89 & 444 & 89 & 444 & 142 & 711 \\
Duty-factor & 0.09 & 0.44 & 0.09 & 0.44 & 0.14 & .71 \\
Nd:GGG disk size (cm) & 6.3 & 2.8 & 11.5 & 5.1 & 9.1 & 4.1 \\
Parasitics gain (log $)$ & 3.8 & 1.7 & 7 & 3.1 & 5.5 & 2.5 \\
Diode output power (MW) & 1.1 & 0.22 & 3.7 & 0.74 & 2.3 & 0.46 \\
Diode cost (M\$) @ 2\$/pk-W & 2.2 & 0.45 & 7.4 & 1.5 & 4.6 & 0.66 \\
Disk weight, + cladding (kg) & 7.5 & 10.3 & 22 & 26.6 & 14.4 & 18.1 \\
\hline
\end{tabular}

Columns with the number of sub-magazines equal to one correspond to conventional HCLs. The last two columns assume a larger temperature swing, $160 \mathrm{C}$ compared to 100 $\mathrm{C}$; this can be realized by decreasing the doping level and increasing the pumping irradiance, as proposed initially by Ray Beach (LLNL, 2000). The blue rows show selected values, while the non-colored rows show calculated values.

The design point for the study below has 10 disks, $6 \mathrm{~cm}$ x $6 \mathrm{~cm}$ x $1.5 \mathrm{~cm}$ each, in the laser cavity, and five two-second sub-magazines. The nominal average power is $100 \mathrm{~kW}$. 


\subsection{Mechanical design}

The objectives for the mechanical/thermal design were:

- Pre-conditioned disks must be available for rapid insertion, $\sim 1 \mathrm{~s}$, into the laser cavity

- Compactness

- Maximum use of off-the-shelf parts (towards rapid prototype)

- Simple, easily manufactured

The design approach is summarized in Table 4.1

Table 4.1: Mechanical design approach

- Modular

- Permits design and optimization of a single module

- lower cost

- more rapid design and analysis

- key issues can be checked at the module level (use multi-pass resonator for extraction demos)

- Internal vacuum

- 1 atmosphere results in excessive OPD after a few pulses. OPD estimate after 6 pulses:

- maximum temperature of boundary layer $\sim 20 \mathrm{C}$

- boundary layer thickness $\sim 2 \mathrm{~cm}=1 / 3-\mathrm{d}$ of cavity height

- results is sharp OPD gradient near top of cavity $\sim 36$ waves (4.8 if air replaced by He)

- Manufacturable

- conventional machine tools

- aluminum when possible

- housing cast or welded

- Disk mounting compatible with optical constraints

- alloy matching disk CTE (Carpenter Glass-Sealing 42-6, 6.5x10-6/C)

- allow mount to locally expand with disk

The study did not consider rapid cooling. The applications addressed are those requiring a finite magazine for the mission duration, such that no cooling is required during the mission. However, the following thermal requirements were imposed:

- the laser disks must be at a known, uniform temperature at the start of an engagement - cooling must be possible in between missions, in times on the order of 10-30 minutes, by edge-cooling. 
Three concepts were developed in significant detail. They are:

- Concept 1: Translated disk matrix

- Concept 2: Swing arm

- Concept 3: "Slide projector"

All concepts are modular, with the number of modules equal to the number of gain elements in the laser cavity. The first two concepts will be discussed briefly. The third concept represents the optimum identified to-date. The designs were performed by W. Griffin using state-of-the-art CAD tools.

\subsubsection{Concept 1: Translated disk matrix}

The first concept, shown in Figure 4.1, has multiple disks in a 2-D matrix. The figure shows a $3 \times 3$ matrix of disks supported in a cooled frame. The matrix support provides cooling of each disk from two edges. Disks are inserted in the laser cavity by transverse translation. Figure 4.2 shows a disk matrix installed in its housing.

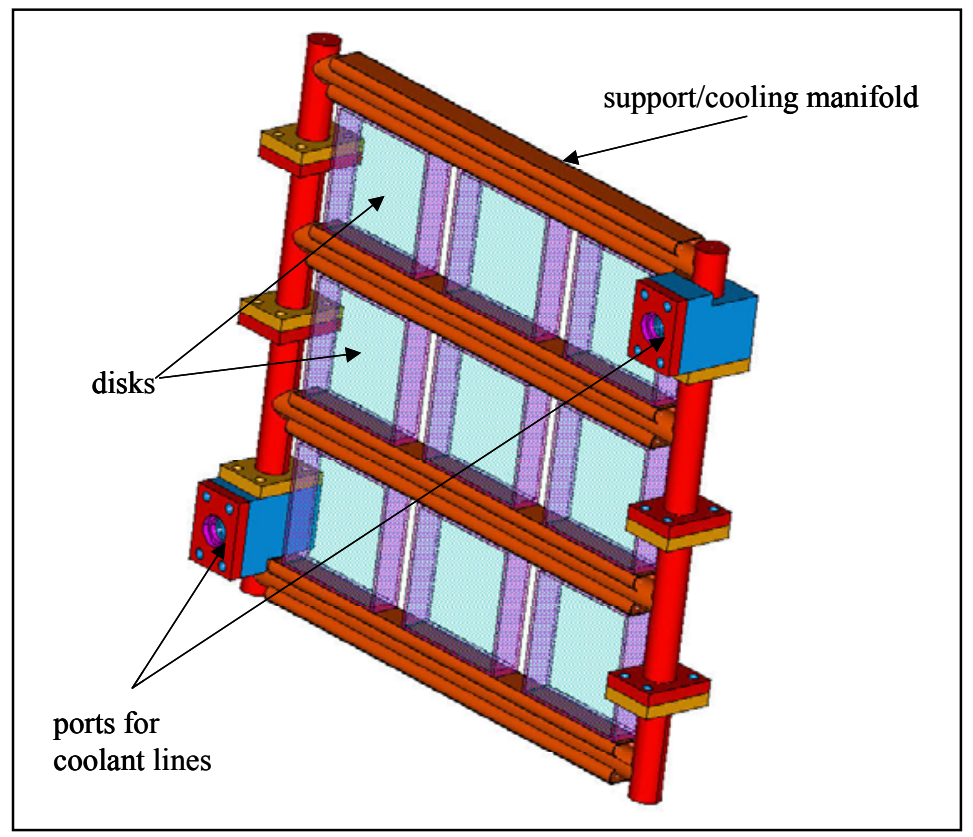

Figure 4.1 Translated disk matrix, 3 x 3 array

Attractive features of this design are conceptual simplicity and good cooling (for slow reset only). However, size is much larger than desired. In addition, the manifold is flexible and may be incompatible with a military environment. 


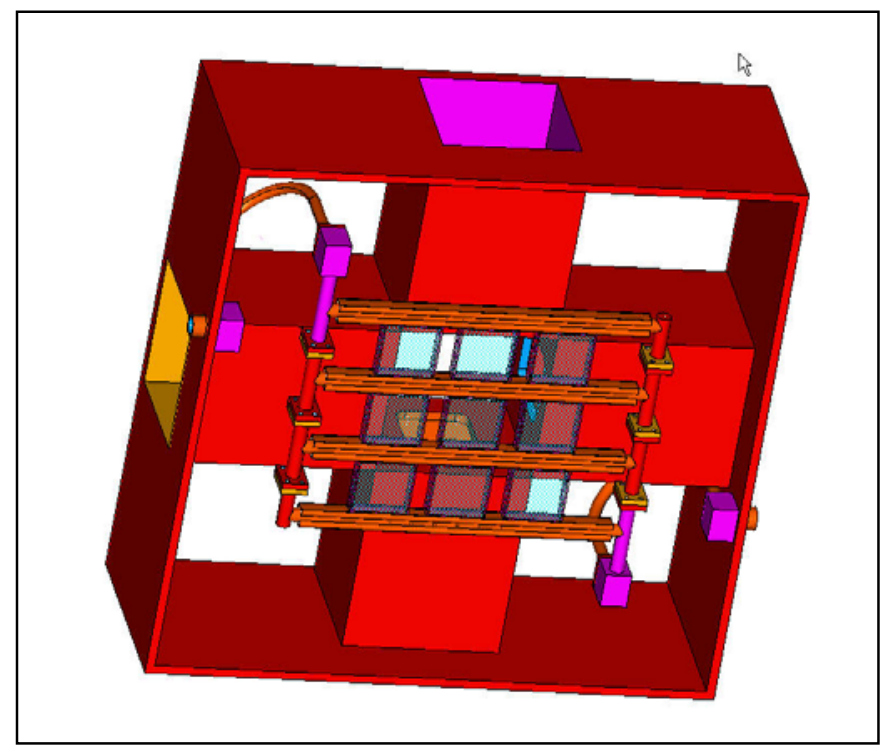

Figure 4.2 Disk matrix installed in its housing

\subsubsection{Concept-2: Swing Arm}

The second concept has disks stowed in a carrier that is translated axially. Disks are moved from the carrier into the laser cavity using a "swing-arm" mechanism. Figure 4.3 shows a cross-section. Figure 4.4 shows a complete module, with one disk deployed in the laser cavity. Figure 4.5 shows dual disk carriers with first slabs deployed; each carrier holds four disks.

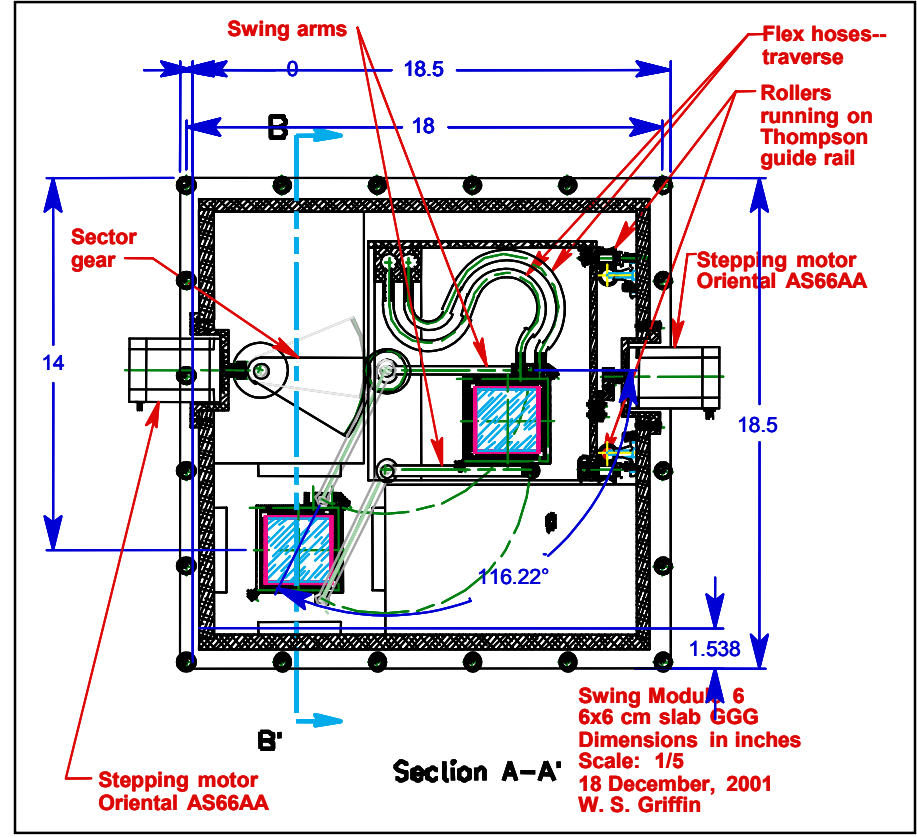

Figure 4.3 Swing arm concept cross section 


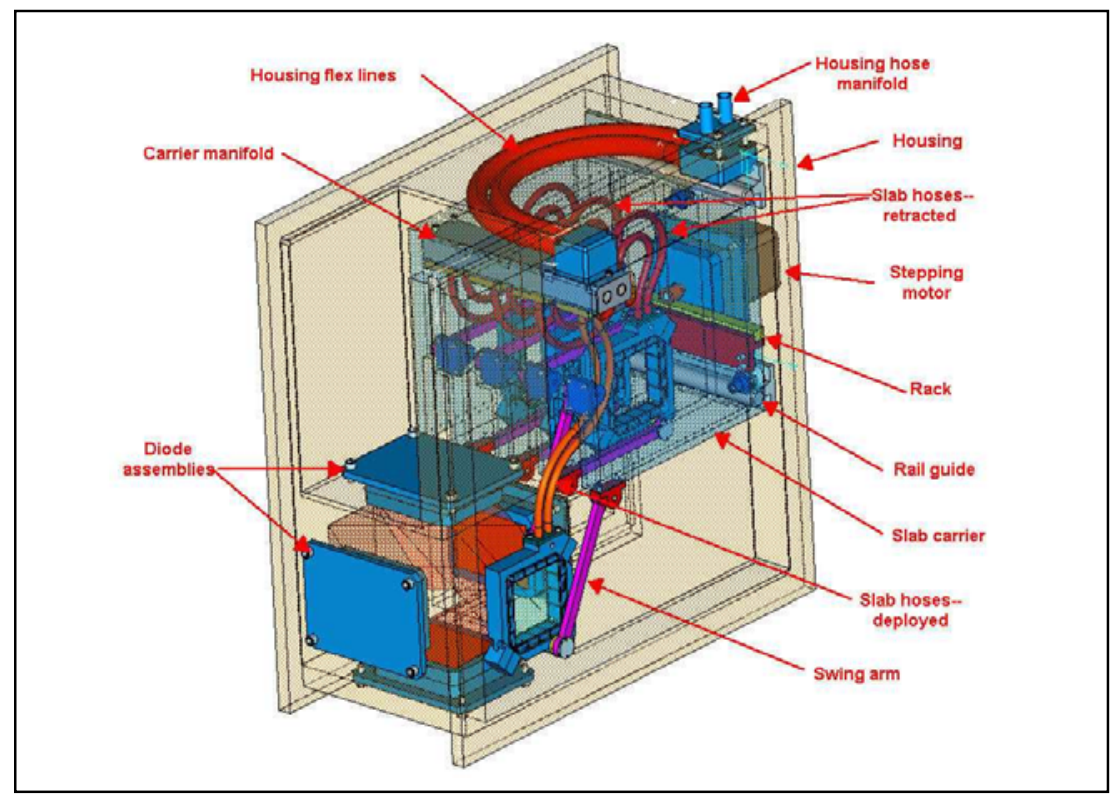

Figure 4.4 Complete module (housing shown translucent)

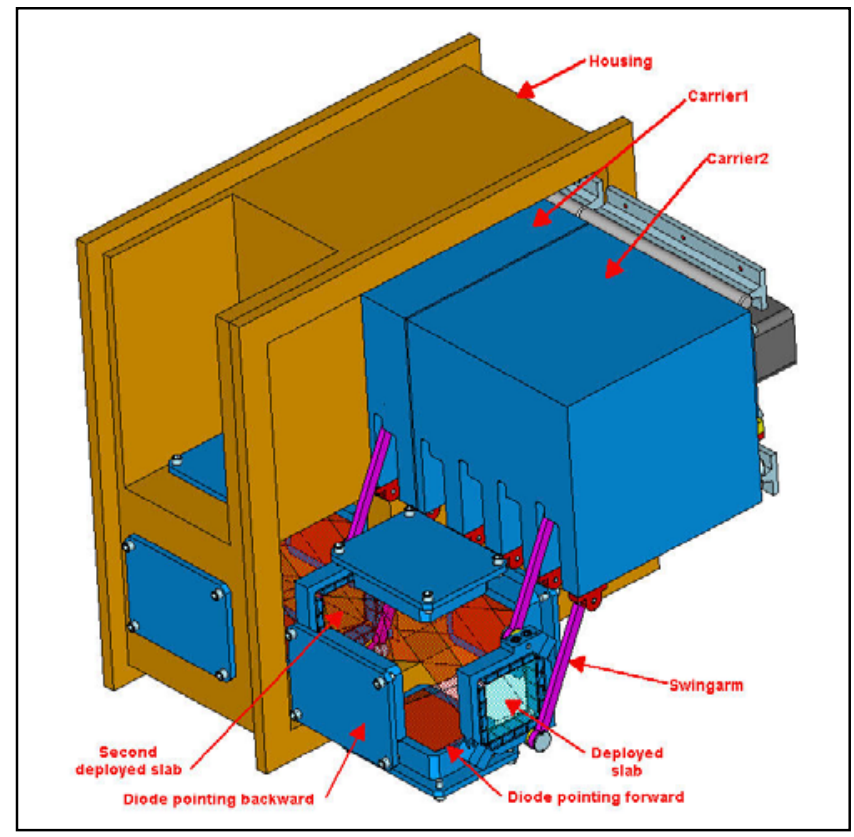

Figure 4.5 Dual disk carriers with first disks deployed

Advantages over concept-1 are:

- Reduced cross section

- Much more rugged and vibration-resistant:

- deployed disks mate into sockets (not shown)

- retracted disks fit securely into slots in carrier

- Improved disk translation: 
- simple axial translation: stepping-motor driving a rack

- but swing-arm actuation requires more work

- Disk mounting and cooling substantially improved

- cooling on 4 edges

- flex-hoses provide good reliability, no rotating fluid seals

However, the system is still larger than desired.

\subsubsection{Concept-3: Slide Projector}

The third concept is based on translation in two dimensions. Carriers are translated axially, similar to concept-2. Disks are translated into/out-of a carrier and into/out-of the laser cavity using a carriage. Figures 4.6-4.8 show visualizations of the approach.

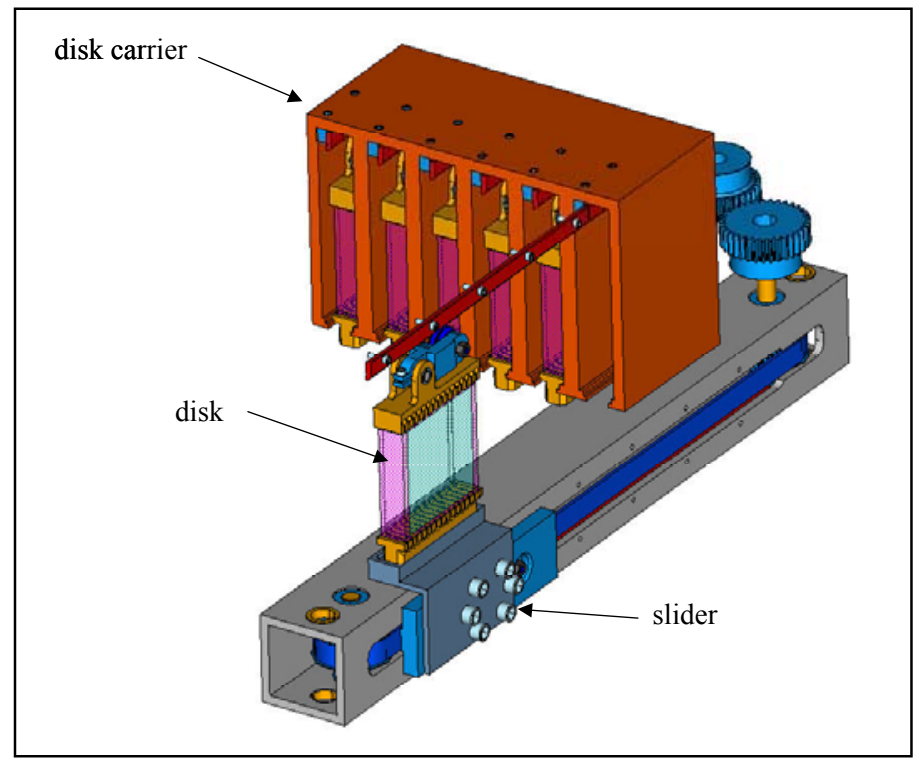

Figure 4.6 Concept visualization: a carriage moves disks between laser cavity and carrier

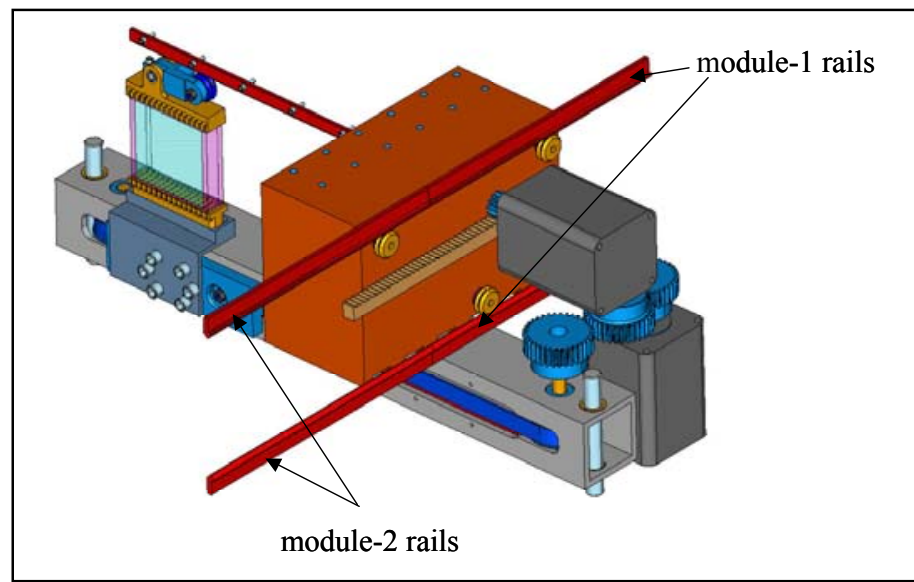

Figure 4.7 Concept visualization: both translations are powered by stepping motors 


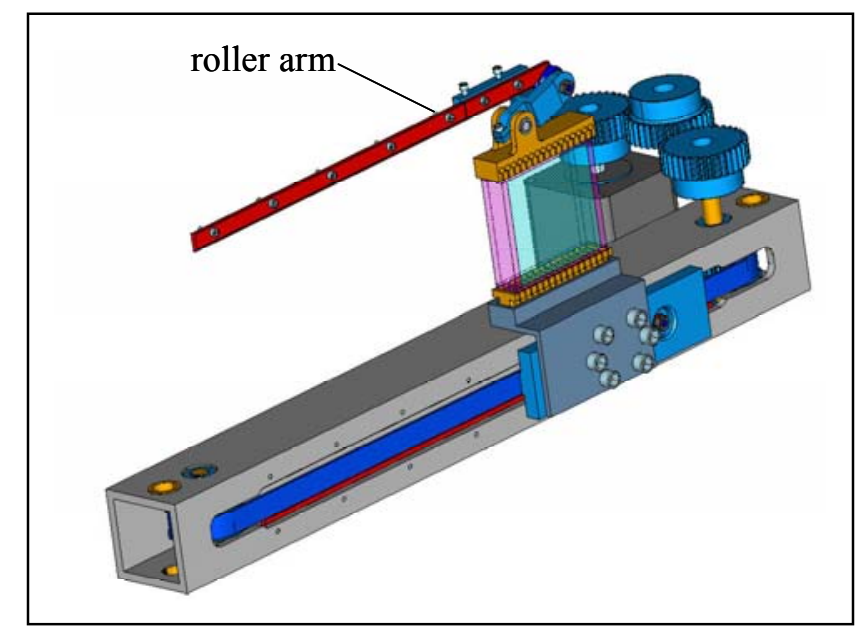

Figure 4.8 Concept visualization: carriage mechanism

Disk carriers ride onto the rails of the adjacent module during translation. Figure 4.9 illustrates the slow-cooling approach. Foam metal is used to thermally interface each disk to a carrier. A cold plate with flex-hoses is bolted to the carrier for thermal control. Flex hoses allow leak-free, low-friction transfer of coolant to the cold plate. Bolt-on manifolds interface the flex hoses to the outside.

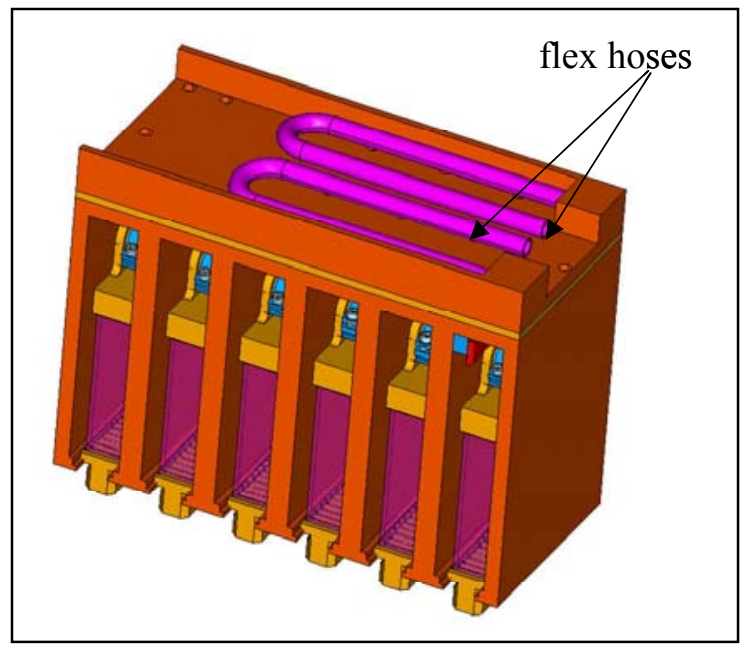

Figure 4.9 Slow-cooling approach

Figure 4.10 shows a complete module. It stores 6 disks (one more could be squeezed in). The module volume is about 1 cubic foot, and its weight $35 \mathrm{~kg}\left(\right.$ density $\left.\sim 1.2 \mathrm{~g} / \mathrm{cm}^{3}\right)$. It should be noted again that these numbers are representative only, and should not be considered to be optimum. 


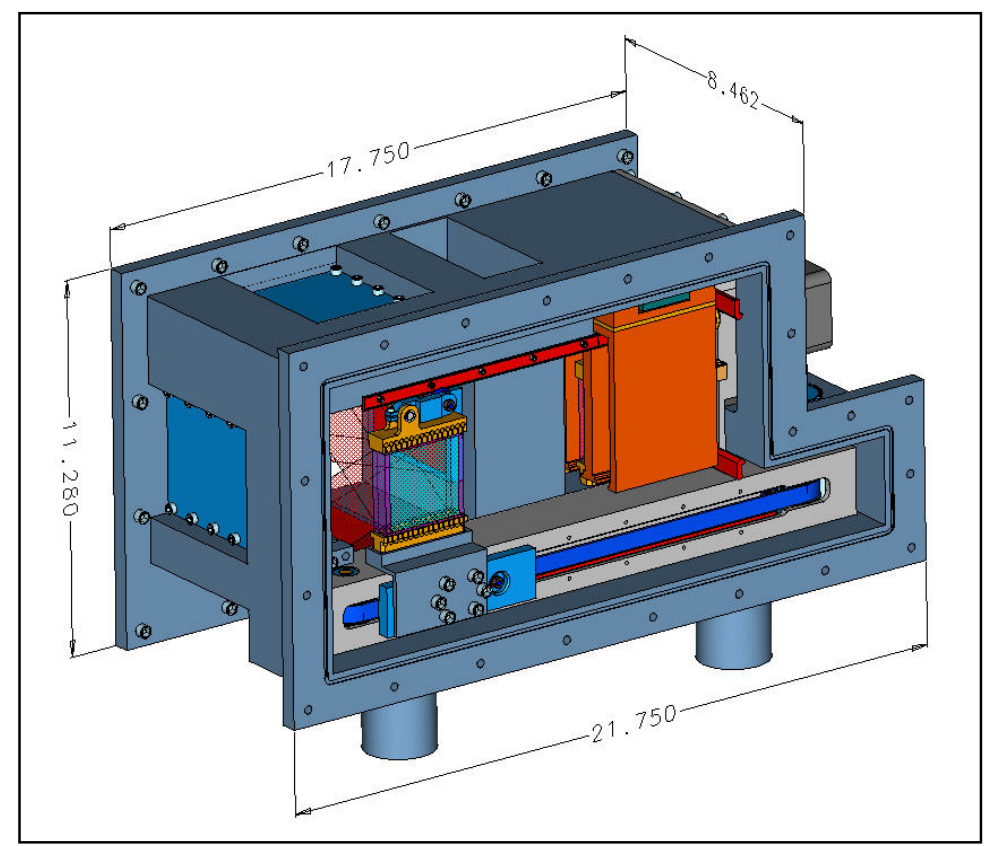

Figure 4.10 Complete module

Advantages of this concept over the second one are:

- a substantial increase in magazine depth, 6-7 disks instead of 4,

- improved mechanization of disk translation,

- maximum use of off-the-shelf hardware. 


\subsection{Electrical Design}

The laser disks are pumped by large diode arrays. The differences with HCLs are:

- higher duty factor, typically $50 \%$ as compared to $10 \%$

- smaller array sizes (due to smaller disk sizes)

Since maximum peak power is not a requirement, the pulse length is typically $1-2 \mathrm{~ms}$ (or longer), compared to $0.5 \mathrm{~ms}$ for an HCL. This allows direct use of batteries, resulting in substantial size/weight savings. Figure 4.10 shows a simplified schematic. Strip-lines connect the diode arrays to the power source. The switches are thermally contacted to the diode cooling water inlet/outlet.

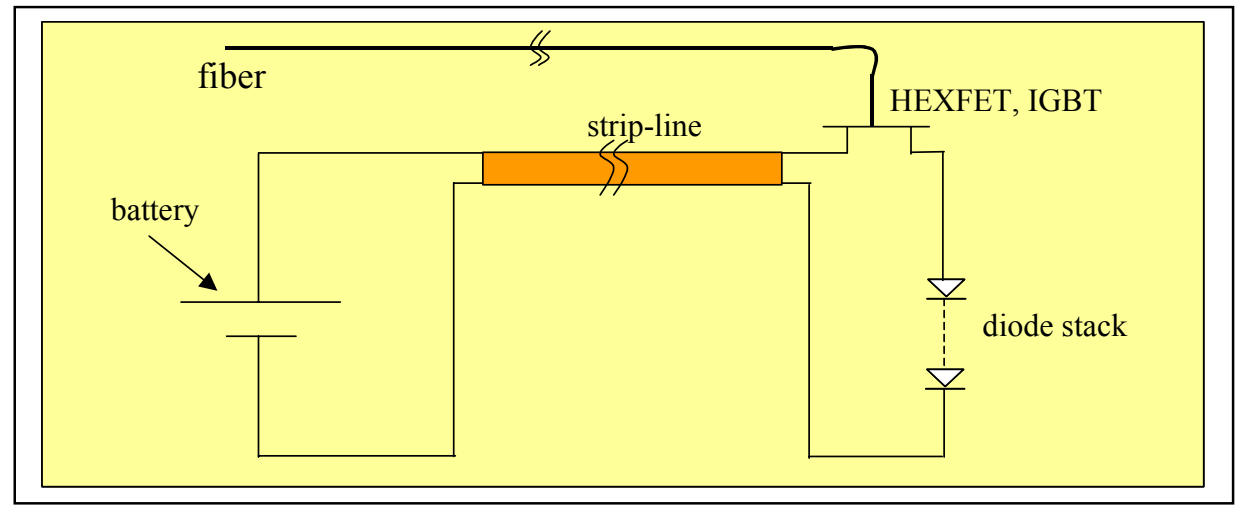

Figure 4.10 Simplified diode driver schematic

\subsection{Hybrid HCL-MSL Power Scaling}

Scaling to significantly larger magazines may be possible using demonstrated crystal sizes on the order of $10 \mathrm{~cm}$. Figure 5.1 shows an architecture for a $1.5 \mathrm{MW}, 30 \mathrm{sec}(45$ MJ) HEL. The laser has three stages, with each stage containing 8 modules and each module containing 8 disks. Approximate design parameters are:

- $\quad \mathrm{E}=1500 \mathrm{~J}(500 \times 3), \mathrm{f}=1 \mathrm{kHz}, \mathrm{t}_{\mathrm{p}} \sim 1 \mathrm{~ms}$

- $\Delta \mathrm{T}=160 \mathrm{C}$

- Disk size: $10.4 \mathrm{~cm}$ x $10.4 \mathrm{~cm} \times 2 \mathrm{~cm}$

- Total disk weight (3 stages): $\sim 550 \mathrm{~kg}$

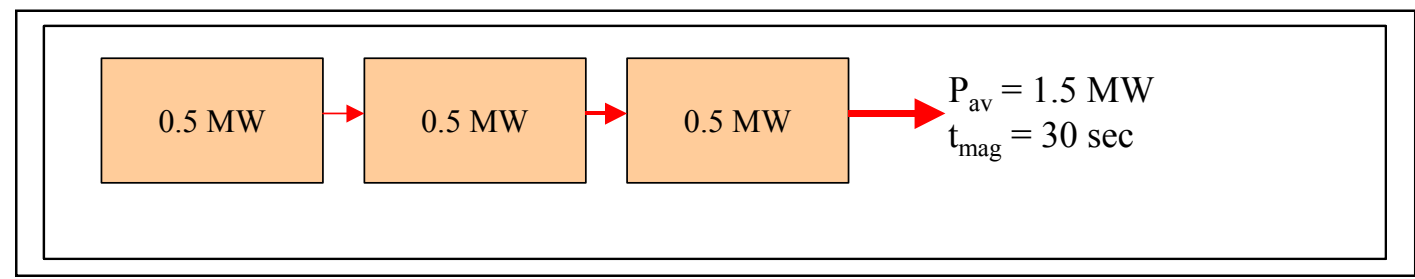

Figure 5.1 Architecture for 1.5 MW, 30s hybrid HCL-MSL 


\subsection{Conclusions}

Hybrid HCL-MSLs have a significant cost advantage over conventional HCLs, i.e. up to a factor of 5-10 in diode cost. Their enabling technology is near-CW diode arrays, and such arrays have already been demonstrated at LLNL using microchannel cooler technology. The Nd:GGG crystal size is smaller than for HCLs, 5-6 cm for a $100 \mathrm{~kW}$ laser, which is within the SOA. Larger disks, $10-11 \mathrm{~cm}$, operated over a temperature swing of about $160 \mathrm{C}$, may allow power scaling to about $0.5 \mathrm{MW}$.

\subsection{Acknowledgements}

The author wants to acknowledge the highly professional consulting help provided in this study by W.S. Griffin of WSG Enterprises and R.L. Anderson of American Control Engineering. Bill generated the mechanical designs shown in this report, while Bob provided the electrical design.

The author also acknowledges numerous stimulating discussions on the hybrid HCLMSL concept with Drs. W. Krupke and W. Sooy, and on the conventional HCL concept with Drs. B. Dane and M. Rotter. 


\subsection{References}

1. G.F. Albrecht, S.B. Sutton, E.V. George, W.R. Sooy, and W.F. Krupke, Solid state heat capacity disk laser, Laser and Particle Beams, 1998, vol. 16, no. 4, pp.605-625.

2. G. Albrecht, E.V. George, W. Sooy and S.B. Sutton, High energy bursts from a solid-state laser operated in the heat capacity limited regime, U.S. patent 5,526,372, 11 June 1996.

3. S. Basu and R.L. Byer, A fixed cavity, moving slab Nd:Glass laser, SPIE Society of Photo-Optical Instrumentation Engineers, 1987, SPIE-Volume 736, pp. 34-37.

4. R.L. Byer and S. Basu, Moving Slab Laser, U.S. Patent 4,833,682, May 23, 1989.

5. J.A. Skidmore et al., Silicon monolithic microchannel-cooled laser diode array, Applied Physics Letters, Vol. 77, Number 1, July 2000.

6. W. Krupke, Optics Communications, 12 (2) 210, 1974.

7. B. Comaskey et al., IEEE JQE 31 (7), 1261, 1995. 